\title{
PROPOSTA DE NOVA CLASSIFICAÇÃO PLUVIOMÉTRICA POR MEIO DA AVALIAÇÃO DOS ÍNDICES DE SEVERIDADE DA SECA NA REGIÃO DE ITAPORANGA-PB
}

\author{
NASCIMENTO, Maxsuel Bezerra do - maxsuel10gba@hotmail.com \\ Universidade Estadual da Paraíba/ UEPB
}

SILVA, Andréia Freitas - andreiafreitassilva@gmail.com

Universidade Estadual da Paraíba/ UEPB

MARTILDES, Jéssica Araújo Leite - jessmartildes@gmail.com

Universidade Estadual da Paraíba/ UEPB

PAIVA, William de - wpaiva461@gmail.com

Universidade Estadual da Paraíba/ UEPB

SANTOS, Laércio Leal dos - laercioeng@yahoo.com.br

Universidade Estadual da Paraíba/ UEPB

Submetido em: 23/07/2020

Aceito para publicação em: 12/03/2021

Publicado em: 06/04/2021

DOI: http://dx.doi.org/10.5380/abclima.v28i0.75405

\begin{abstract}
RESUMO: As secas interferem nas áreas ambiental, agrícola e/ou econômica. A seca é devido à falta ou redução da precipitação no decorrer do tempo fora da normalidade. Existem diferentes índices de secas com metodologias distintas que podem se associar a uma determinada região de forma mais específica. Sendo assim, o objetivo desse trabalho é aplicar uma nova abordagem na classificação das secas na região de Itaporanga-PB a partir da correlação entre os seguintes índices de seca: Índice de Porcentagem Normal (IPN), Método Decis (MD) e o Índice de Anomalia de Chuva (IAC). Para esse fim, uma série pluviométrica histórica foi utilizada, no qual foi possível avaliar dados quantitativos em escala mensal e anual, cujo resultado determina a intensidade das secas no decorrer do tempo. Os dados pluviométricos mensais correspondem ao período de 1994 a 2019 fornecidos pela Agência Executiva de Gestão das Águas do Estado da Paraíba (AESA). Os resultados obtidos demonstram uma resposta satisfatória na delimitação dos períodos chuvosos e secos da região, de modo a projetar cenários futuros para o gerenciamento dos recursos hídricos na área estudada.
\end{abstract}

PALAVRAS-ChAVE: Períodos, Escassez hídrica, Variabilidade da chuva, Índice de Seca

PROPOSAL FOR A NEW PLUVIOMETRIC CLASSIFICATION THROUGH THE EVALUATION OF DROUGHT SEVERITY INDICES IN THE REGION OF ITAPORANGA-PB

ABSTRACT: Droughts interfere in the environmental, agricultural and / or economic areas. Drought is due to the lack or reduction of exclusion over time to normality. There are different drought rates with different methodologies that can be associated with a specific region in a more specific way. Thus, the objective of this work is to apply a new approach to the classification of droughts in the region of Itaporanga-PB based on the correlation between the following drought indices: Normal Percentage Index (IPN), Decis Method (MD) and the Index of Rain Anomaly (IAC). For this purpose, a historical rainfall series was used, in which it was possible to evaluate quantitative data on a monthly and annual scale, the result of which determines the intensity of droughts over time. Monthly rainfall data for the period 1994 to 2019 provided by the Executive Water Management Agency of the State of Paraíba (AESA). The results obtained demonstrate a satisfactory 
response in the delimitation of the rainy and dry periods in the region, in order to project future scenarios for the management of water resources in the studied area.

KEYWORDS: Periods, Water scarcity, Rain variability, Drought index

PROPUESTA DE UNA NUEVA CLASIFICACIÓN PLUVIOMÉTRICA A TRAVÉS DE LA EVALUACIÓN DE ÍNDICES DE SEVERIDAD DE SEQUÍA EN LA REGIÓN DE ITAPORANGA$P B$

RESUMEN: Las sequías interfieren en las áreas ambiental, agrícola y / o económica. La sequía se debe a la falta o reducción de la exclusión a lo largo del tiempo hasta la normalidad. Existen diferentes tasas de sequía con diferentes metodologías que pueden asociarse con una región específica de una manera más específica. Así, el objetivo de este trabajo es aplicar un nuevo enfoque a la clasificación de las sequías en la región de Itaporanga-PB basado en la correlación entre los siguientes índices de sequía: Índice de Porcentaje Normal (IPN), Método de Decis (MD) y el Índice de Anomalía de lluvia (IAC). Para ello se utilizó una serie histórica de precipitaciones, en la que fue posible evaluar datos cuantitativos a escala mensual y anual, cuyo resultado determina la intensidad de las sequías en el tiempo. Datos mensuales de precipitación para el período 1994 a 2019 proporcionados por la Agencia Ejecutiva de Gestión del Agua del Estado de Paraíba (AESA). Los resultados obtenidos demuestran una respuesta satisfactoria en la delimitación de los períodos lluvioso y seco en la región, con el fin de proyectar escenarios futuros para la gestión de los recursos hídricos en el área estudiada.

PALABRAS CLAVE: períodos, escasez de agua, variabilidad de la lluvia, índice de sequía

\section{INTRODUÇÃO}

As secas produzem cenários desastrosos em várias áreas da sociedade, seja na esfera ambiental, agrícola e econômica. A seca refere-se à falta/redução de chuva no decorrer do tempo em determinado local, cujo seu início é de difícil detecção. Deste modo, seus impactos são múltiplos e severos, podendo causar danos diretos e indiretos sobre os determinantes ambientais, econômicos, sociais (MAGALHÃES, 2016; HAGENLOCHER et al., 2019; MEZA et al., 2019).

Assim, a seca é um fenômeno natural que, dependendo da sua magnitude, pode ser desastroso (PETRUNGARO \& HORA, 2019). Outrossim, os impactos provenientes das secas, podem desestabilizar a subsistência de populações que vivem em terras áridas ou semiáridas, podendo levar a uma crise de desenvolvimento humano invisível e ao processo de migração (UNCCD, 2014; VERNER, 2016).

De acordo com Costa e Silva (2017), o monitoramento das secas pode ser realizado via índices climáticos, os quais caracterizam os períodos secos e chuvosos, para entender a variabilidade climática de uma localidade, estado ou região.

A seca que pode ser classificada como: meteorológicas, as que podem ser expressas pela falta de chuvas e durabilidade da secura de um determinado ambiente. Através de um período constante, de um mês ou mais, durante o qual a precipitação está abaixo dos valores de chuva média para o período. Seca (NORONHA et al., 2016).

Seca agrícola, pela falta de água no solo para o desenvolvimento das plantas, no qual torna o suprimento de água às culturas insuficiente para repor as perdas por evapotranspiração (DUARTE et al., 2018).

Seca hidrológica, que está relacionada diretamente à diminuição dos mananciais, no qual deixa-os abaixo do nível médio. Além disso, está 
relacionada com uma deficiência no volume de água disponível, incluindo lençol freático, reservatórios e rios (DUARTE et al., 2018).

Já a seca socioeconômica, que se interliga a produção humana, que se interliga diretamente com a produção agrícola. De acordo com Fernandes et al. (2009), inclui os impactos diretos e indiretos na produção agrícola e outras atividades econômicas.

Logo, todas as secas estão interligadas diretamente aos fatores ambientais e pela variabilidade climática. Portanto, se destacam alguns dos sistemas atmosféricos que interferem de forma significativa na precipitação pluvial (chuva) do Nordeste brasileiro (NEB), são eles: a Zona de Convergência Intertropical (ZCIT), Vórtices Ciclônicos em Altos Níveis (VCAN), distúrbios nos ventos alísios, linhas de instabilidade (LI), sistemas frontais (SF) no sul de NEB e Zona de Convergência do Atlântico Sul - (ZCAS) - (NÓBREGA e SANTIAGO, 2014; ASSIS et al. 2015; PALHARINI e VILA,2017).

A Paraíba é um dos estados do NEB que proporciona uma das maiores variabilidade espaço-temporal das chuvas, por exemplo, a região do Cariri/Curimataú é a parte do Estado da Paraíba que apresenta um baixo índice pluviométrico de 300 a 500 mm, no Sertão e Alto Sertão em torno de 700 a 900 $\mathrm{mm}$ de chuva, no Brejo e Agreste, com chuvas que variam de 700 a $1.200 \mathrm{~mm}$, e o Litoral proporciona as maiores medias de chuva entre 1.200 a 1.600 mm.anual ${ }^{-1}$ (Francisco et al., 2015).

Segundo Lima (2016), a seca é uma anomalia que não pode ser evitada e, por isso, deve-se sempre agir de forma preventiva. Neste sentido como existem diferentes índices de seca com distintas metodologias, faz-se necessária uma avaliação do desempenho sobre qual índice apresenta melhor desempenho na região. E importante realizar intercomparação entre os índices para se ter um resultado satisfatório. Deste modo, neste trabalho foram utilizados o, Método dos Decis (MD) - (Gibbs e Maher 1967), Índice de Anomalia de Chuva (IAC) (Rooy 1965) que foi readaptado por Araújo et al. (2009) e o Índice de Porcentagem Normal (IPN) - (Cunha, 2008).

Portanto, o objetivo deste trabalho é aplicar uma nova classificação do índice de seca aos dados da série pluviométrica histórica da região de Itaporanga no estado da Paraíba, com intuito de classificar os dados quantitativos em escala mensal e anual, a partir da correlação dos métodos de IPN e MD e com o método de IAC sem alteração, a fim de identificar a intensidade no decorrer dos anos estudados nesta pesquisa.

\section{MATERIAL E METODOS}

\section{ÁREA DE ESTUDO}

A área de estudo compreende a antiga microrregião de Itaporanga, que pertencia à mesorregião do Sertão Paraibano, localizado no Estado da Paraíba (Figura 1). De acordo com Instituto Brasileiro de Geografia e Estatística (IBGE) a população é estimada, para o ano de 2019, em 85.863 habitantes. Possui uma área total de $3.053,939 \mathrm{~km}^{2}$ e é dividida em onze municípios: Boa Ventura, Conceição, Curral Velho, Diamante, Ibiara, Itaporanga, Pedra Branca, Santa Inês, Santana de Mangueira, São José de Caiana e Serra Grande. 


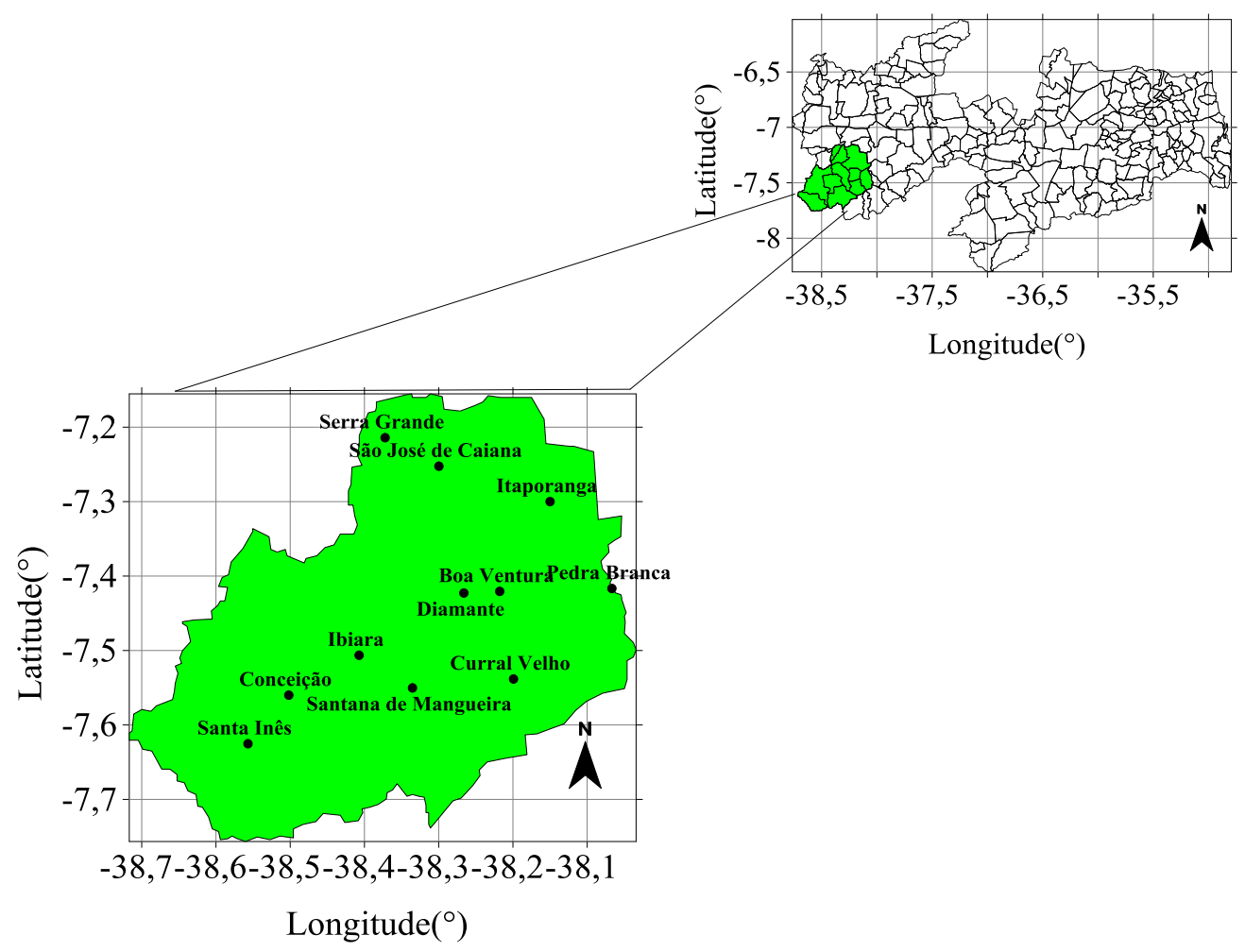

Figura 1 - Área de estudo com a localização dos 11 municípios da Região de Itaporanga, em relação ao estado da Paraíba.Fonte: Autoria própria.

\section{DADOS PLUVIOMÉTRICOS}

Os dados pluviométricos utilizados correspondem à série pluviométrica mensal histórica do período de 1994 a 2019, fornecidos pela Agência Executiva de Gestão das Águas do Estado da Paraíba (AESA). Com base nos dados pluviométricos de 12 pontos meteorológicos localizados na região de estudo, sendo possível determinar a chuva na escala mensal e anual.

\section{ÍNDICES DE SECA}

A partir das séries temporais históricas foram realizados os cálculos dos três índices de seca, com o auxílio de planilhas eletrônicas do Microsoft Excel 2016.

\section{MÉTODO DOS DECIS - MD}

Para o cálculo do MD agrupou-se as médias para cada período analisado em ordem crescente e a partir da distribuição de frequência acumulada dividiuse a série em dez partes iguais (decis). A classificação deste método é baseada em Gibbs e Maher (1967). Segundo Lima (2016), serve para determinar a probabilidade de ocorrência de um evento e também para definir de forma qualitativa a anomalia climática. 


\section{ÍNDICE DE ANOMALIA DE CHUVA - IAC}

A análise da duração dos períodos secos e úmidos foi realizada por meio do cálculo do IAC, (FREITAS, 2004 e 2005), obtido a partir das equações e de acordo com a Tabela 1:

IAC $=3\left[\begin{array}{l}(N-\bar{N}) \\ (\bar{M}-\bar{N})\end{array}\right]:$ Para anomalias positivas
IAC $=-3\left[\begin{array}{l}(N-\bar{N}) \\ (\bar{M}-\bar{N})\end{array}\right]:$ Para anomalias negativas

(equação 1)

(equação 2)

Sendo:

$\mathrm{N}=$ Precipitação mensal atual $(\mathrm{mm})$;

$\bar{N}=$ Precipitação média mensal da série histórica $(\mathrm{mm})$;

$\bar{M}=$ Média das dez maiores precipitações mensal da série histórica $(\mathrm{mm})$;

$\bar{X}=$ Média das dez menores precipitações mensais da série histórica $(\mathrm{mm})$.

Tabela 1 - Classes de intensidades do índice de anomalia de chuva (IAC)

\begin{tabular}{ccc}
\hline & FAIXA DO IAC & CLASSES DE INTENSIDADE \\
\hline Índice & De 4 acima & Extremamente úmido \\
De anomalia & 2 a 4 & Muito úmido \\
De chuva & 0 a 2 & Úmido \\
(IAC) & 0 a -2 & Seco \\
& -2 a -4 & Muito seco \\
& De -4 abaixo & Extremamente seco \\
\hline
\end{tabular}

Fonte: Araújo et al. (2009)

Logo, este método foi utilizado em diversos trabalhos nos últimos anos para a definição pluviométricas das secas e das características de determinada região (NÓBREGA et al. 2016, MANIÇOBA et al. 2017; NASCIMENTO et al. 2020).

\section{ÍNDICE DE PORCENTAGEM NORMAL - IPN}

O IPN proporciona simplicidade em seus cálculos e pode ser empregado para diferentes escalas temporais, alterando na maioria das vezes entre um mês ou um conjunto de meses até um ano (NDMC, 2020)

Para o cálculo do IPN Cunha (2008), que expressa, em porcentagem, a razão entre a precipitação atual e a precipitação normal (média de 26 anos) de uma região, utilizou-se a Equação 3.

$I P N=\frac{P A T U A L}{P N O R M A L}$

em que,

IPN = Índice de Porcentagem Normal;

PATUAL = Precipitação em um dado local $(\mathrm{mm})$;

PNORMAL $=$ Precipitação média do período analisado $(\mathrm{mm})$. 


\section{NOVA PROPOSTA DE CLASSIFICAÇÃO DA SEVERIDADE DE SECAS E ADAPTAÇÕES PARA OS ÍNDICES DO IPN E MD}

Considerou-se que a análise dos resultados dos diversos índices de seca é de difícil compreensão, neste sentido procurou-se avaliar, o grau de severidade da seca com base nas classificações propostas para cada um dos índices avaliados. A utilização das classificações visou verificar a frequência das diferentes intensidades de seca dos índices quando aplicados na região de Itaporanga-PB.

Destaca-se que esse procedimento foi modificado também, de acordo com a escala de tempo, muitos autores sugerem que sejam acima de 30 anos de dados (Lima, 2016; Silva et al. 2020;), nesta nova classificação, se demostrou que com os dados existente no trabalho é possível esta adaptação.

Os índices de IPN e do MD sofreram uma adaptação em suas classes para dá uma maior visibilidade no estudo, por meio de uma padronização no sistema de classificação em seis intensidades, de acordo com o IAC e ficarem no mesmo parâmetro, são eles: Extremamente Úmido (EU), Muito Úmido (MU), Úmido (U), Seco (S), Muito Seco (MS) e Extremamente Seco (ES).

No Quadro 1 está exposta a classificação do IPN sugerida por Cunha (2008) e a classificação proposta no presente trabalho, na qual foram adotados diferentes intervalos numéricos para cada uma das classes de intensidade de seca. Nota-se que o número de classes aumentou, para ficar de acordo com o método do IAC proposto por Araújo et al. (2009) e do mesmo modo estar em um parâmetro de mesma escala e identificar adequadamente as anomalias pluviométricas para a região.

Quadro 1 - Relação entre os valores de IPN de Cunha (2008) e o proposto pelos autores.

\begin{tabular}{|l|l|l|l|}
\hline \multicolumn{2}{|c|}{ Cunha (2008) } & \multicolumn{2}{c|}{ Proposto } \\
\hline IPN & $\begin{array}{l}\text { INTENSIDADE } \\
\text { DE SECA }\end{array}$ & IPN & $\begin{array}{l}\text { INTENSIDADE DE } \\
\text { SECA }\end{array}$ \\
\hline IPN $\geq 0,85$ & Normal & IPN $\geq 1,50$ & Extremamente úmido \\
\hline $0,75 \leq$ IPN $<0,85$ & Seca moderada & $1,25 \leq$ IPN 1,50 & Muito úmido \\
\hline $0,50 \leq$ IPN $<0,75$ & Seca severa & $1,00 \leq$ IPN 1,25 & Úmido \\
\hline IPN $<0,50$ & Seca extrema & $0,75 \leq$ IPN $<1,00$ & Seco \\
\hline & & $0,50 \leq$ IPN $<0,75$ & Muito seco \\
\hline & & IPN $<0,50$ & Extremamente seco \\
\hline
\end{tabular}

Fonte: Autoria própria

Já no Quadro 2 apresenta-se a classificação das secas segundo Gibbs e Maher (1967) e, também, a proposta. 
Quadro 2 - Relação entre os valores de MD e o proposto pelos autores.

\begin{tabular}{|c|c|c|c|}
\hline \multicolumn{2}{|c|}{ Gibbs e Maher (1967) } & \multicolumn{2}{|c|}{ Proposto } \\
\hline MD & $\begin{array}{c}\text { INTENSIDADE } \\
\text { DE SECA }\end{array}$ & MD & INTENSIDADE DE SECA \\
\hline $10-9$ & Muito Úmido & 10 & Extremamente úmido \\
\hline $8-7$ & Úmido & $9-8$ & Muito úmido \\
\hline $6-5$ & Próximo ao Normal & $7-6$ & Úmido \\
\hline $4-3$ & Seco & $5-4$ & Seco \\
\hline $2-1$ & Muito Seco & $3-2$ & Muito seco \\
\hline & & 1 &
\end{tabular}

Fonte: Autoria própria

De acordo com o modelo proposto para o MD no Quadro 2, é adicionado novas classes considerando os valores extremos máximo e mínimo, e retirando a normalidade proposto por Gibbs e Maher (1967). Logo, esses novos valores propostos, nos possíveis resultados, precisaram de uma atenção maior quando os resultados demostrarem os seus extremos positivo ou negativo.

\section{CLASSIFICAÇÃO DOS ÍNDICES DE SECA NA ESCALA DE TEMPO MENSAL E ANUAL}

Após análise do banco de dados e de sua classificação nas duas escalas de tempo analisadas (mensal e anual) e das metodologias abordadas pelos índices utilizados na Tabela 2.

Tabela 2 - Classificação da intensidade de seca dos índices IPN, MD, IAC e limites de classe propostos.

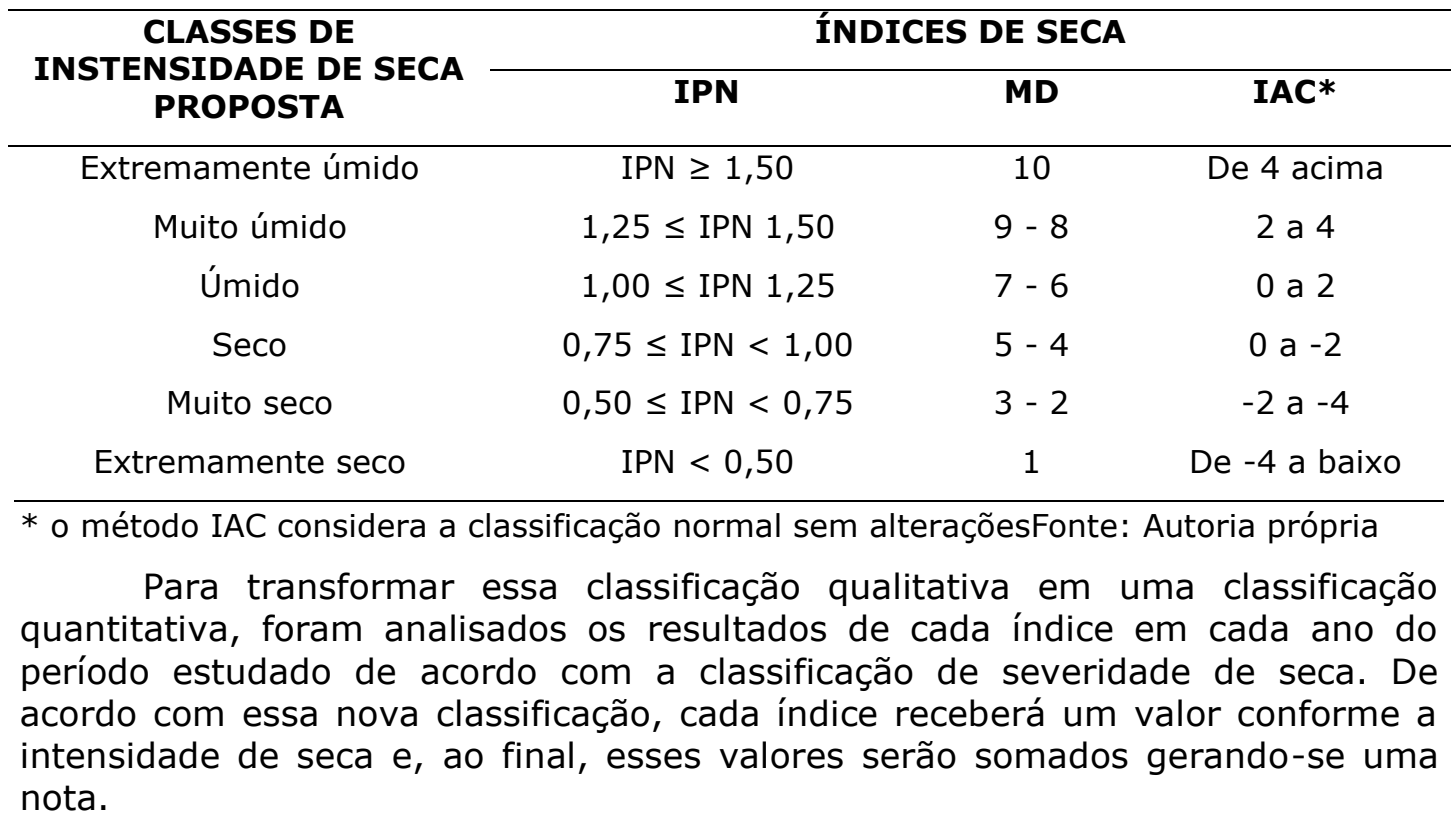


Portanto, a classificação pode variar de "Emergencial de Seca", até "Emergência de Chuva" entre os seis índices. Neste sentido, serão aplicados esses parâmetros dessa classificação na escala mensal e anual, para uma compreensão dos resultados.

Para criar o sistema de classificação de secas, levou-se em consideração que todos os índices possuem a mesma intensidade de seca proposto, conforme explicado na Tabela 2. Logo esse valor global foi utilizado para determinar a intensidade de todos os meses e anos, do período adotado no trabalho.

Através de uma correlação entre os índices de seca analisados, foi proposta uma nova classificação para a situação de seca de cada método em estudo. Portanto cada índice possui sua metodologia, porém, no fim ambos possuem o mesmo valor. Através disso, foi criado a classificação baseada nos índices de seca, que está para evidenciar de uma maneira mais dinâmica o seu resultado final, correlacionando os três índices.

Logo, foram delimitados valores para os índices de seca no qual se estabeleceu com base nos possíveis somatórios de notas e recebendo o nome de Índice de Avaliação Pluviométrica - IAP (Quadro 3) que podem ser observadas algumas combinações de classes de intensidade de seca e sua classificação.

Essa classificação do IAP foi definida pelas possíveis combinações dos Índices IPN, MD e IAC, que nos possíveis cenários podem apresentar respostas similares, ou diferentes entre ambos. Assim, desta forma no conjunto de dados analisados, dividindo-o em seis classes conforme a Tabela 2, que varia de "Extremamente seco" a "Extremamente úmido", observou que esse parâmetro foi dividido em vinte notas.

Portanto, para cada nota foi estabelecido um nome na proposição da nova classificação, no qual cada classe tem seus valores determinados justamente pelo conjunto de possíveis resultados apresentados pelos índices da Tabela 2. Logo, o Índice de Avaliação Pluviométrica proposto, foi dividido em seis classes recebendo os nomes de Emergência de Seca, Alerta de Seca, Normal de Seca, Normal de Chuva, Alerta de Chuva e Emergência de Chuva, no qual ainda foram subdivididos em notas. 
Quadro 3 - Proposição de combinações das classes de intensidade de seca, para determinação da nota do Índice de Avaliação Pluviométrica.

\begin{tabular}{|c|c|c|c|c|}
\hline Índice de Avaliação Pluviométrica & Nota & IPN & MD & IAC \\
\hline \multirow[t]{2}{*}{ Emergência de Seca } & 1 & ES & ES & ES \\
\hline & 2 & ES & ES & MS \\
\hline \multirow{4}{*}{ Alerta de Seca } & 3 & ES & MS & MS \\
\hline & 4 & MS & MS & MS \\
\hline & 5 & MS & MS & $\mathrm{S}$ \\
\hline & 6 & ES & MS & $\mathrm{S}$ \\
\hline \multirow{4}{*}{ Normal de Seca } & 7 & MS & $\mathrm{S}$ & $\mathrm{S}$ \\
\hline & 8 & $\mathrm{~S}$ & $\mathrm{~S}$ & $\mathrm{~S}$ \\
\hline & 9 & $\mathrm{~S}$ & $\mathrm{~S}$ & $U$ \\
\hline & 10 & MS & $\mathrm{S}$ & $\mathrm{U}$ \\
\hline \multirow{4}{*}{ Normal de Chuva } & 11 & $\mathrm{~S}$ & $U$ & 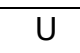 \\
\hline & 12 & $U$ & $U$ & $\mathrm{U}$ \\
\hline & 13 & $U$ & $\mathrm{U}$ & $\mathrm{MU}$ \\
\hline & 14 & $\mathrm{~S}$ & $\mathrm{U}$ & $\mathrm{MU}$ \\
\hline \multirow{4}{*}{ Alerta de Chuva } & 15 & $\mathrm{U}$ & $\mathrm{MU}$ & $\mathrm{MU}$ \\
\hline & 16 & MU & $\mathrm{MU}$ & $\mathrm{MU}$ \\
\hline & 17 & $\mathrm{MU}$ & $\mathrm{MU}$ & EU \\
\hline & 18 & $\mathrm{U}$ & MU & EU \\
\hline \multirow[t]{2}{*}{ Emergência de Chuva } & 19 & MU & EU & EU \\
\hline & 20 & EU & EU & EU \\
\hline
\end{tabular}

$\mathrm{ES}=$ Extremamente Seco; $\mathrm{MS}=$ Muito Seco; $\mathrm{S}=\mathrm{Seco} ; \mathrm{U}=$ Úmido; $\mathrm{MU}=$ Muito Úmido; $\mathrm{EU}=$ Extremamente Úmido.Fonte: Autoria própria

Neste sentido, cada classe com as respectivas notas, foram delimitadas da seguinte forma (Quadro 3), a Emergência de Seca que possui notas 1 e 2 levou em consideração, que quando ocorrer três resultados iguais, ou seja ES em todos os índices, será atribuído a nota 1 . Logo a nota 2, leva-se em consideração quando dois, dos três índices apresentarem com ES e um MS.

Já a classe do Alerta de Seca, foram atribuídas quatro notas que vão de 3 a 6, no qual foi possível destacar que: quando se possui a nota 3 leva-se em consideração que os índices, apresentaram dois deles MS e um ES; para a nota 4 todos os índices apresentam-se com MS; já para a classificação da nota 5 levou-se em consideração que os mesmos apresentaram dois MS e um S; e pôr fim a nota 6 é atribuída quando os índices possuem variações entre eles sendo ES, MS e S, neste caso atribui a nota de classificação a variável que está no meio.

A classificação da classe Normal de Seca, levou-se em conta as notas que vai de 7 a 10: no qual é possível notar que a classe 7 ela é atribuída quando se possui dois S e um MS; já a nota 8 é quando todos os índices se apresentam com S; além disso, a nota 9 é quando se apresenta com dois $S$ e um $U$; em seguida a nota 10 é quando os índices apresentam valores diferentes como MS, S e U.

Para a classe Normal de Chuva, que vai entre as notas 11 a 14: a nota 11 ela ocorrera quando possuir dois $U$ e um $\mathrm{S}$; a 12 quando todos os índices 
forem U; a nota 13 será composta por duas U e uma MU; já a nota 14 será utilizada quando aparecer entre eles S, U e MU.

A penúltima classe que é a de Alerta de Chuva, no qual apresenta quatro notas que vão da 15 a 18: no qual a nota 15 é composta quando dois índices apresentarem como $\mathrm{MU}$ e um $\mathrm{U}$; a 16 quando todos os índices se apresentarem com MU; a nota 17 quando apresentar dois MU e um EU; e a nota 18 quando aparecer em índices diferentes as variáveis U, MU e EM.

Por fim, a última classe denominada Emergência de Chuva, que possui duas notas: sendo elas a 19, que será empregada quando ocorrer duas EU e um MU; e a nota 20 quando todos os índices se apresentarem como EU.

Deste modo, ao aplicar o sistema de classificação de seca na escala de tempo mensal e anual proposto, buscou-se assim verificar a periodicidade das anomalias mais extremas e um possível padrão existente na série temporal.

Logo, o sistema de classificação permitirá examinar as condições de seca e identificar assim os meses e anos com índices mais extremos, sendo eles os de "Emergência de Chuva ou de Seca", e observar a sua relação na sequência cronológica do estudo, verificando sua variação.

\section{RESULTADOS E DISCUSSÃO}

Ao analisar a Figura 2 é possível observar dois períodos distintos, um período de cinco meses chuvoso e outro com sete meses secos. Ao analisar os dados obtidos, foi verificado que o período chuvoso ocorreu entre os meses de janeiro a maio, sendo o mês de março o mais representativo, no qual atinge precipitação média de $196 \mathrm{~mm}$. Em contrapartida, o período seco ocorre entre os meses de junho a dezembro, tendo setembro como o mês mais seco com 2,2 $\mathrm{mm}$.

De acordo com Nascimento et al. (2018), as chuvas nessa região semiárida são caracterizadas por sua concentração em apenas poucos meses do ano, podendo ocorrer chuvas de grande intensidade. Nesse período, pode ocorrer além de $70 \%$ de toda a precipitação anual, logo os outros meses abaixo da média são caracterizados por seca, com precipitações mensais médias abaixo de $20 \mathrm{~mm}$. 


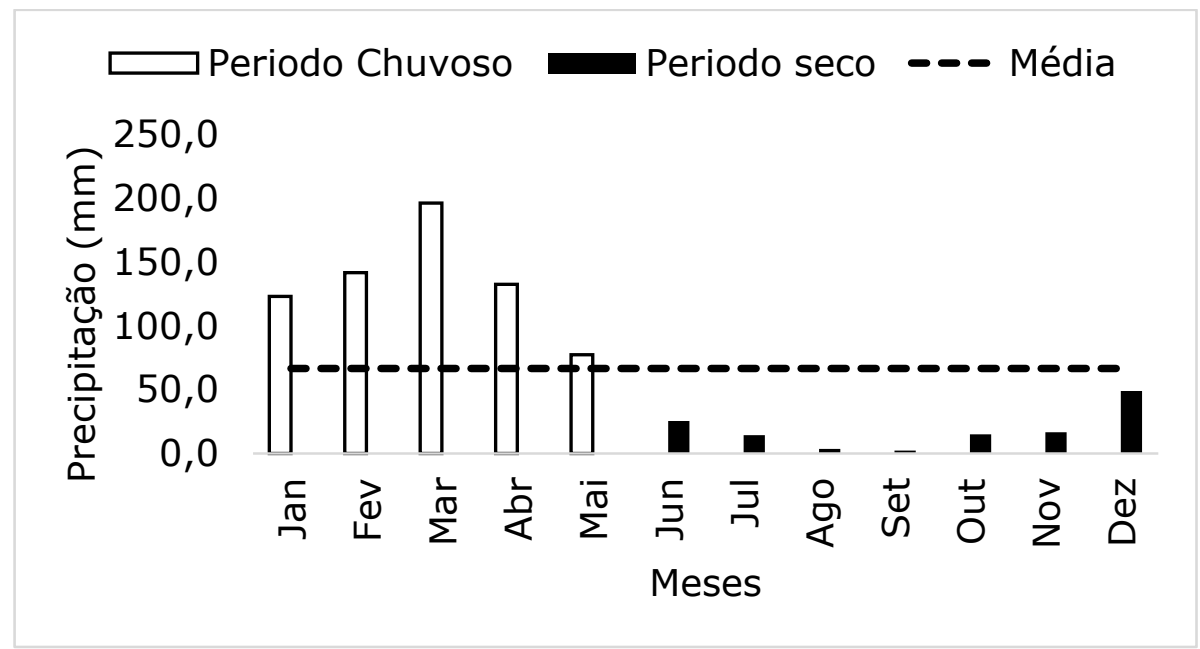

Figura 2 - Pluviograma de Chuva mensal $(\mathrm{mm})$ dos 26 anos da Região de Itaporanga. Fonte: Autoria própria

Os resultados dos índices IPN, MD e IAC são apresentados em duas diferentes escalas de tempo (mensal e anual) para a região de Itaporanga-PB. Portanto, de acordo com o Quadro 4, o IPN apresentou variações, tanto no resultado mínimo como no máximo em seus extremos. Observa-se que os quatro primeiros meses do ano foram bem acima da normalidade apresentando seis meses com extremos de seca.

Já o MD e IAC ofereceram maior constância nos seus resultados, assim todas as classes demonstradas e posteriormente mais homogênea, entre os métodos analisados, na escala mensal, os MD e IAC, apresentaram mais adequação a realidade do que o IPN, visto que ele constitui quatro dados compatíveis com o IAC e o MD observados no Quadro 2. Por outro lado, o MD e o IAC apresentam 9 resultados em comum, demonstrando dessa forma ser mais eficaz nessa área dentre os métodos, pois obterão resultados mais próximos.

Quadro 4 - Classificação mensal dos Índices de Seca na escala de 1994 a 2019 conforme a nova classificação proposta.

\begin{tabular}{|c|c|c|c|c|c|c|c|c|c|c|c|c|}
\hline Índices & Jan & Fev & Mar & Abr & Mai & Jun & Jul & Ago & Set & Out & Nov & Dez \\
\hline \multirow{2}{*}{ IPN } & 1,85 & 2,13 & 2,96 & 1,99 & 1,17 & 0,38 & 0,22 & 0,05 & 0,03 & 0,22 & 0,25 & 0,74 \\
\cline { 2 - 13 } & EU & EU & EU & EU & MU & ES & ES & ES & ES & ES & ES & MS \\
\hline \multirow{2}{*}{ MD } & 8 & 10 & 10 & 9 & 7 & 5 & 2 & 1 & 10 & 3 & 4 & 6 \\
\cline { 2 - 12 } & MU & EU & EU & MU & U & S & MS & ES & ES & MS & S & U \\
\hline \multirow{2}{*}{ IAC } & 2,2 & 2,7 & 4,7 & 2,4 & 0,4 & $-2,1$ & $-2,7$ & $-3,3$ & $-3,3$ & $-2,7$ & $-2,5$ & $-1,0$ \\
\cline { 2 - 11 } & MU & MU & EU & MU & U & MS & MS & MS & MS & MS & MS & S \\
\hline
\end{tabular}

$\mathrm{ES}=$ Extremamente Seco; $\mathrm{MS}=$ Muito Seco; $\mathrm{S}=\mathrm{Seco} ; \mathrm{U}=$ Úmido; $\mathrm{MU}=$ Muito Úmido; $\mathrm{EU}=$ Extremamente Úmido.Fonte: Autoria própria

De acordo com o Quadro 4, constata-se que cada mês possuiu sua particularidade nos diferentes índices, destacando sua proximidade entre um ou mais índices, seguindo aproximadamente a mesma classe, demostrando assim um sinal de severidade nas informações atribuídas para a região, no qual foi possível delimitar o Índice de Avaliação Pluviométrica mensal. 
É possível verificar que o período da série histórica estudada apresentou 13 anos variando entre meses chuvosos e secos, delimitados segundo a nova escala proposta (Quadro 3), onde destaca-se o mês de maio como um mês de transição entre o período mais húmido e o mais seco, e os meses de janeiro a abril sendo o período mais úmido e entre os meses de junho a dezembro o período mais seco, com uma pequena anomalia para o MD onde no mês de dezembro foi úmido.

Através dos métodos utilizados, e da classificação proposta para os índices, que a área em estudo apresenta dois períodos distintos (Figura 3), o primeiro possuindo seus máximos de precipitação entre os meses de fevereiro e março e os mínimos de precipitação nos meses de agosto e setembro, ficando demonstrado, os meses de maior e menor contribuição pluviométrica e consequentemente os meses de maior carência hídrica que representam resultados idênticos ao pluviograma temporal da Figura 2.

Portanto, a água possui papel fundamental no desenvolvimento de toda a sociedade, desempenhando funções não apenas ecológicas, mas também econômicas e sociais (CALADO et al. 2020). Diante dos resultados obtidos, torna-se necessário um gerenciamento dos recursos hídricos locais baseados no conhecimento dos períodos de maior e menor contribuição hídrica da área em estudo, com políticas que priorizem a preservação de água nos períodos mais secos e o período de utilização mais abundante dos mananciais no período chuvoso.

Além disso, o incentivo as atividades agrícolas típicas da região, favorecendo dessa forma o desenvolvimento econômico e a permanência do homem no campo. Corroborando com Aquino \& Oliveira (2013), que estas informações contribuem para o planejamento agrícola, e as práticas de controle da produção, além de auxiliar na identificação das fragilidades climáticas.

\section{Índice de Avaliação Pluviométrica Mensal}

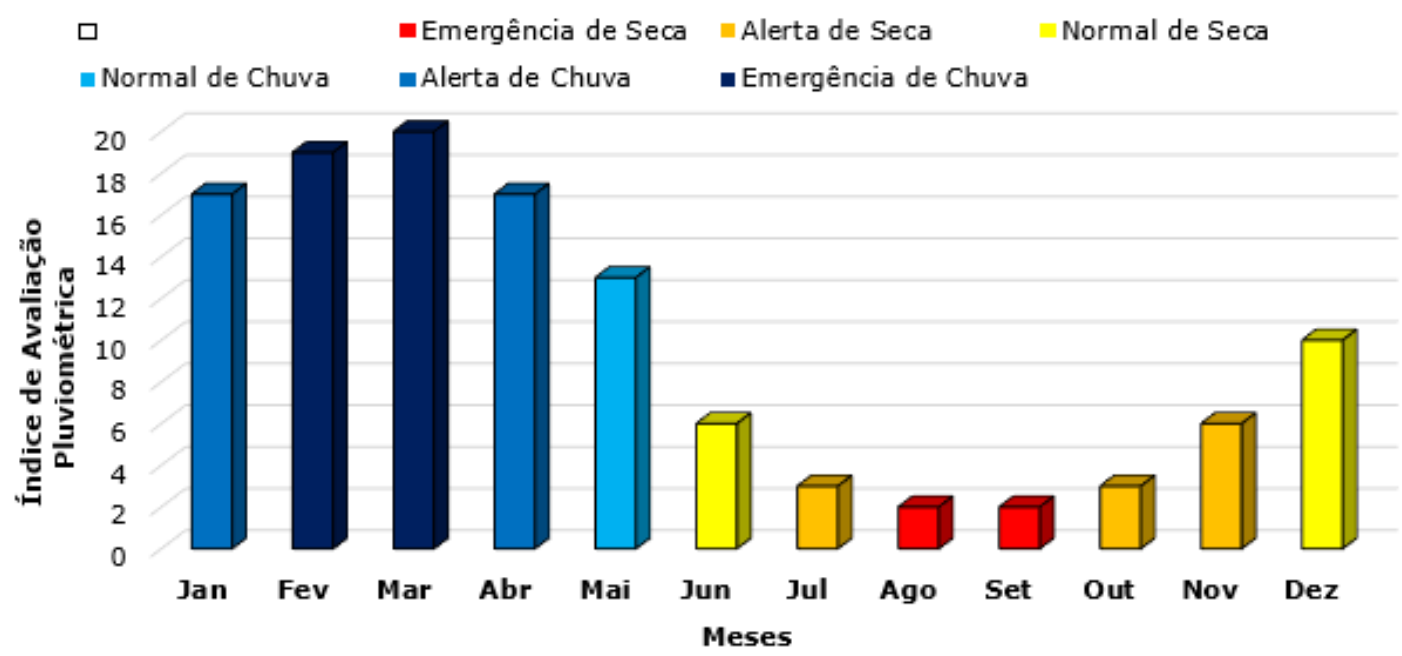

Figura 3 - Índice de Avaliação Pluviométrica mensal da Região de Itaporanga conforme a nova classificação proposta. Fonte: Autoria própria

A análise anual da intensidade da seca, correspondente ao Quadro 3, no qual apresenta os parâmetros de cada índice, onde foi possível observar que 
para o IPN os anos extremos positivos aparece em apenas 1 ano, para o MD e o IAC 3 anos, aonde é possível observar que para todos os índices a condição de anos classificados como extremo seco foram os mais encontrados.

Por outro lado, o número de anos na utilização dos três índices na escala anual, demonstrou que os valores mais encontrados estão entre seco e úmido, ou seja, demonstra uma constante alternância entre períodos chuvosos e secos. Assim Nascimento et al, (2019), diz que em séries pequenas ou grandes de precipitação, o primordial é a alternância entre períodos secos e chuvosos, pois assim se notará um equilíbrio sem prejudicar as atividades no meio.

De acordo com o Quadro 5, dentre os três índices de seca analisado no estudo, o índice IPN apresentou 18 dados similares com os outros índices, enquanto que o MD apresentou 20 dados similares, e o IAC apresentou uma variação mais notável que os demais índices obtendo 24 dados similares com os outros índices, mostrando a sua importância no estudo proposto.

Diante disso, o IAC se destaca com melhores resultados na área estudada, porém os demais índices também apresentaram resposta positivas, principalmente o MD, como na análise mensal. Portanto, a utilização dos índices é que se pode chegar a um resultado positivo ou negativo para toda região, destacando que o conjunto dos índices foi de extrema importância para delimitar os parâmetros. Dessa forma, o estudo dos índices com a nova classificação proposta apresentou resultados promissores podendo ser aplicados a outras regiões. 
Quadro 5 - Classificação anual dos Índices de Seca na escala de 1994 a 2019 conforme a nova classificação proposta.

\begin{tabular}{|c|c|c|c|c|c|c|}
\hline \multirow{3}{*}{$\begin{array}{c}\text { Ano } \\
1994\end{array}$} & \multicolumn{6}{|c|}{ Índices de Seca } \\
\hline & \multicolumn{2}{|c|}{ IPN } & \multicolumn{2}{|c|}{ MD } & \multicolumn{2}{|c|}{ IAC } \\
\hline & 1,34 & $\mathrm{MU}$ & 10 & EU & 3,4 & $\mathrm{MU}$ \\
\hline 1995 & 0,80 & $\mathrm{~S}$ & 2 & MS & 2,7 & $\mathrm{MU}$ \\
\hline 1996 & 1,09 & U & 7 & $\mathrm{U}$ & 0,7 & $\mathrm{U}$ \\
\hline 1997 & 1,08 & U & 6 & $U$ & 0,5 & $U$ \\
\hline 1998 & 0,50 & MS & 1 & ES & $-5,5$ & ES \\
\hline 1999 & 0,96 & S & 5 & $\mathrm{~S}$ & $-0,7$ & S \\
\hline 2000 & 1,23 & $\bar{U}$ & 9 & MU & 2,2 & MU \\
\hline 2001 & 0,82 & $\mathrm{~S}$ & 3 & MS & $-2,2$ & MS \\
\hline 2002 & 1,10 & U & 7 & $U$ & 0,8 & $U$ \\
\hline 2003 & 1,02 & U & 6 & $\mathrm{U}$ & 0,0 & $\mathrm{~S}$ \\
\hline 2004 & 1,26 & $\mathrm{MU}$ & 9 & $\mathrm{U}$ & 3,7 & $\mathrm{MU}$ \\
\hline 2005 & 0,95 & S & 4 & $\mathrm{~S}$ & $-0,9$ & $\mathrm{~S}$ \\
\hline 2006 & 1,32 & MU & 10 & EU & 3,2 & MU \\
\hline 2007 & 0,80 & $\mathrm{~S}$ & 3 & MS & $-2,3$ & MS \\
\hline 2008 & 1,57 & EU & 10 & EU & 5,9 & EU \\
\hline 2009 & 1,30 & MU & 9 & $\mathrm{MU}$ & 3,0 & MU \\
\hline 2010 & 0,88 & $\mathrm{~S}$ & 4 & $\mathrm{~S}$ & $-1,3$ & $\mathrm{~S}$ \\
\hline 2011 & 1,16 & U & 8 & MU & 1,4 & U \\
\hline 2012 & 0,46 & ES & 1 & ES & $-5,6$ & ES \\
\hline 2013 & 1,09 & $U$ & 7 & $\mathrm{U}$ & 0,7 & $\mathrm{U}$ \\
\hline 2014 & 0,82 & S & 3 & MS & $-2,1$ & MS \\
\hline 2015 & 0,75 & S & 2 & MS & $-2,8$ & MS \\
\hline 2016 & 0,61 & MS & 1 & ES & $-4,3$ & ES \\
\hline 2017 & 0,96 & $S$ & 5 & $\mathrm{~S}$ & $-0,7$ & $\mathrm{~S}$ \\
\hline 2018 & 1,18 & $\bar{U}$ & 8 & MU & 1,6 & $\bar{U}$ \\
\hline 2019 & 0,95 & $S$ & 5 & $S$ & $-0,3$ & $\mathrm{~S}$ \\
\hline
\end{tabular}

$\mathrm{ES}=$ Extremamente Seco; $\mathrm{MS}=$ Muito Seco; $\mathrm{S}=\mathrm{Seco} ; \mathrm{U}=$ Úmido; $\mathrm{MU}=$ Muito Úmido; $\mathrm{EU}=$ Extremamente Úmido.Fonte: Autoria própria

Da mesma forma, que foram utilizados para se padronizar o estudo mensal, também foi proposta a analise anual, de forma a se compreender melhor o estudo proposto anual, dos períodos de cheia ou seca na região de Itaporanga, conforme a Figura 4, que demonstra a análise anual, que os anos 
apresentaram em sua maioria uma situação de Índice de Avaliação Pluviométrica Anual conforme a classificação proposta.

Portanto, a área de estudo possui segundo a nova classificação proposta conforme o Quadro 3, todas as variações possíveis, apresentando 13 anos chuvosos, sendo classificados como NORMAL DE CHUVA (1996, 1997, 2002, 2003, 2011, 2013 e 2018), ALERTA DE CHUVA (1994, 2000, 2004, 2006 e 2009) e EMERGÊNCIA DE CHUVA (2008). E por outro lado, apresentou 13 anos secos classificados em NORMAL DE SECA (1995, 1999, 2005, 2010, 2017 e 2019), ALERTA DE SECA (2001, 2007, 2014 e 2015) e EMERGÊNCIA DE SECA (1998, 2012 e 2016).

\section{Índice de Avaliação Pluviométrica Anual}

\begin{tabular}{|c|c|c|}
\hline$\square$ & - Emergência de Seca & a Alerta de Seca \\
\hline Normal de Chuva & - Alerta de Chuva & - Emergência d \\
\hline
\end{tabular}

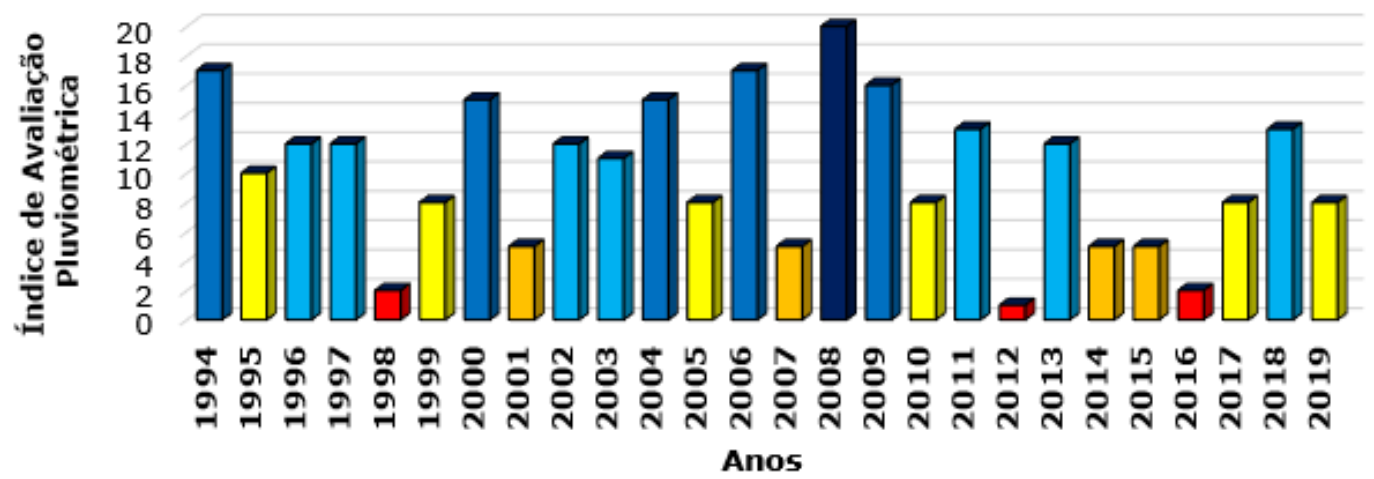

Figura 4 - Índice de Avaliação Pluviométrica Anual da Região de Itaporanga conforme a nova classificação proposta. Fonte: Autoria própria

Assim, através desses resultados é notório observar dentro das escalas mensais e anuais que a escala anual possui uma maior variação para a área em estudo. Se uma correlação for realizada entre os anos classificados como Normal, seja ele considerado chuvoso ou seco, obtém-se um somatório de 13 anos ou $50 \%$, e podemos classifica-los como estáveis e com pouco impacto significativo. Já os anos classificados como "Alerta de Chuva ou Seca", apresentaram juntos cerca de 9 anos ou $34,6 \%$ enquanto que os anos de "Emergência de Seca ou Chuva" apresentaram apenas 4 anos, com 15,4\%, porem é nesses anos onde uma maior atenção deve ser realizada.

Com essa delimitação entre os anos de "Alerta de Chuva e Seca", destaca-se períodos de grandes impactos, tanto econômico, social e ambiental, em uma perspectiva de que as chuvas em excesso, trazem consigo problemas em grandes centros urbanos como, inundações, riscos de desastres ambientais, perca da lavoura e danos na saúde, caso não tenha uma gestão adequada dos recursos hídricos ou falta de conhecimento dos períodos em análise.

Por outro lado, as secas intensas provocam, além da pouca produtividade na agricultura, um aumento na temperatura, danos à saúde humana, mortalidade de animais, êxodo rural, dentre outros. Assim sendo, é notório dizer 
que as secas no semiárido são episódios naturais, não excepcionais (NOBREGA et al. 2016).

Ainda assim, os anos classificados como "Emergência" sofreram grandes impactos, principalmente com o índice de seca mais intenso. Nota-se que os anos de 1998 e 2012 possuem similaridades e seguem uma variação normal, sendo classificados como "emergência", porém, no ano anterior a eles, foram considerados com uma alta pluviosidade.

Logo esses anos não apresentaram tantos impactos sendo classificados como emergência porem, no ano anterior a eles, foram considerados com uma alta pluviosidade, não apresentando assim, um período com tantos impactos, quando comparados aos demais. Já, o ano de 2016 foi o inverso, nota-se que os dois anos anteriores a ele apresentava alerta de seca, ou seja, com pluviosidade bem abaixo do esperado, e no ano de 2016 esse fator aumentou, chegando a um índice extremamente baixo.

Destaca-se ainda que, nos anos de 1998, 2012 e 2016 classificados em seca, podem ter apresentados impactos significativos, principalmente nas atividades locais, consequências do aumento da temperatura e na diminuição dos recursos hídricos, que afetam a quantidade de água disponível nos reservatórios da região (SILVA et al. 2018)

Por fim, os resultados obtidos contribuem para os gestores locais, agricultores e agências ambientais do estado, um conhecimento amplo dos períodos de maior escassez e de maior abundância dos recursos hídricos de forma a permitir proposições de políticas públicas que possibilitem estratégias de convivência do homem do campo e no interior para a melhor convivência em ambos os períodos distintos e dessa forma venham a projetar cenários futuros para o gerenciamento dos recursos hídricos.

Assim sendo, estes cenários podem ser previstos pelo geoprocessamento, através de levantamento de dados da sequência cronológica de uma determinada região, o que possibilita ver a questão da vulnerabilidade em que a região se encontra e prospectar cenários futuros. Além dessa projeção também existe modelos computacionais e matemáticos como o método de Redes neurais que estima cenários futuros e detecta riscos.

\section{CONCLUSÕES}

A região de Itaporanga-PB tem dois períodos distintos de chuva, um chuvoso entre de janeiro a maio, e um seco entre junho a dezembro.

O uso dos índices de secas é eficiente na delimitação dos períodos secos e chuvosos na região de Itaporanga-PB. Destaque para os índices MD e o IAC na avaliação dos extremos de chuva.

O Índice de Avaliação Pluviométrica Mensal e Anual, foram de grande respaldo para a região estudada, onde demonstrou características e delimitações de cada mês e ano.

Conclui-se, que os resultados obtidos contribuem para os gestores locais, agricultores e agencias ambientais do estado, um conhecimento amplo dos períodos de maior escassez e de maior abundância de recursos hídricos. 
Por fim, possibilita estratégias de convivência do homem do campo para a melhor convivência em ambos os períodos distintos e dessa forma venham a projetar cenários futuros para o gerenciamento dos recursos hídricos.

\section{REFERÊNCIAS BIBLIOGRÁFICAS}

ARAÚJO, L. E.; MORAES NETO, J. M.; SOUSA F. A. S. Análise climática da bacia do rio Paraíba - Índice de Anomalia de Chuva (IAC). Revista de Engenharia Ambiental, v.6, n.3, p.508-523, 2009.

Aquino, C.M.S., Oliveira, J.G.B. Emprego do Método de Thornthwaite e Mather (1955) para Cálculo do Balanço Hídrico Climatológico do Núcleo de Degradação de São Raimundo NonatoPiauí. Revista Brasileira de Geografia Física v.6, p.7990, 2013.

ASSIS, J.M.O.; SOUZA, W.M.; SOBRAL, M.C.M. Climate analysis of the rainfall in the lower-middle stretch of the São Francisco river basin based on the rain anomaly index. Revista Brasileira de Ciências Ambientais, v. 2, p. 188-202, 2015.

CALADO, T. O.; E. A. T. Marques, E. A. T.; Sobral, M. C. Planos diretores na articulação da gestão de recursos hídricos com o uso do solo no entorno de reservatórios. Revista Brasileira de Geografia Física, v. 13, n. 3, p. 958-972, 2020.

COSTA, J. A.; DA SILVA, D. F. Distribuição espaço-temporal do Índice de anomalia de chuva para o Estado do Ceará. Revista Brasileira de Geografia Física, v. 10, n. 4, p. 1002-1013, 2017.

CUNHA, R. L. A. Definição de cenários de referência para avaliação dos impactos das secas. Dissertação (Mestrado Integrado em Engenharia Civil), Faculdade de Engenharia da Universidade do Porto, Porto. 2008, $147 \mathrm{f}$.

Duarte, J. G. P.; Farias, A. A.; Sousa, F. A. S.; Souza, J. T. A.; Ramos, M. M. Q. Secas e Impactos na Agropecuária no Município de Campina Grande - PB. Revista Brasileira de Meteorologia, v. 33, n. 2, 289-297, 2018

FRANCISCO, P. R. M; MEDEIROS, R.M; SANTOS, D; MATOS, R. M. Classificação Climática de Köppen e Thornthwaite para o Estado da Paraíba. Revista Brasileira de Geografia Física V. 08 N. 04, 1006-1016, 2015.

FERNANDES, D.S.; HEINEMANN, A.B.; PAZ, R.L.; AMORIM, A.O. \& CARDOSO, A.S. 2009. Índices para a quantificação da seca. Santo Antônio de Goiás: Embrapa Arroz e Feijão, 48p. Disponível em: Acesso em: 20 ago. 2020.

GIBBS, W. J.; MAHER, J. V. Rainfall deciles as drought indicators. Melbourne: Commonwealth of Australia, 1967. 84 p. (Bureau of Meteorology. Bulletin, 48).

HAGENLOCHER, M.; MEZA, I.; ANDERSON, C.C.; MIN, A. Drought vulnerability and risk assessments: state of the art, persistent gaps, and research agenda. Environ. Res. Lett, v.14, 2019.

IBGE - Instituto Brasileiro de Geografia e Estatística. Contagem da população 2019. Brasília: IBGE, 2019. s.p. Disponível em: http://www.ibge.gov.br/cidadessat/index.php. Acesso em: 20 de Julho de 2018. 
LIMA, R. P. C. Avaliação de Índices de severidade de seca na Bacia Do Rio Doce visando ao desenvolvimento de Sistema de Classificação de Secas. 2016. 98p. Dissertação de Mestrado em Engenharia Agrícola -Universidade Federal de Viçosa, Minas Gerais. 2016.

MAGALHÃES, A. Vida e seca no Brasil. In: DE NYS, E.; ENGLE, N.L.; MAGALHÃES, A.R. (orgs.). Secas no Brasil: política e gestão proativas. Brasília, DF: Centro de Gestão e Estudos Estratégicos- CGEE; Banco Mundial, 2016. 292 p.

MANIÇOBA, R. M.; SOBRINHO, J. E.; GUIMARÃES, Í. T.; JUNIOR. E. G. C.; SILVA. T. T. F.; ZONTA, J. H. Índice de anomalias de chuva para diferentes mesorregiões do Estado do Rio Grande do Norte. Revista Brasileira de Geografia Física, v. 10, n. 4, p. 1110-1119, 2017.

MEZA, I.; HAGENLOCHER, M.; NAUMANN, G.; VOGT, J.; FRISCHEN, J. Drought vulnerability indicators for global-scale drought risk assessments. Publications Office of the European Union, Luxembourg, 2019.

NASCIMENTO, J. M.; FRADE, T. G.; SILVA, R. M. Modelagem da resposta do escoamento em uma bacia do semiárido da Paraíba utilizando o modelo SWAT. Revista Brasileira de Geografia Física, v. 11, n. 3, p. 1137-1150, 2018.

NASCIMENTO, M. B. DO; BANDEIRA, M. M.; ARAÚJO, L. E. DE. Variabilidade climática da precipitação do município de Areia/Paraíba, Brasil. Gaia Scientia, v. 13, n. 3, p.24-37, 2019.

NASCIMENTO, M. B.; ALMEIDA, N. V.; ARAUJO, L. E. Análise da variabilidade da precipitação pluviométrica na microrregião de Umbuzeiro, Paraíba. Revista Brasileira de Climatologia, v. 26, p.233-248, 2020.

NDMC - National Drought Mitigation Center, University of Nebraska, Lincoln, Nebraska, EUA. http://drought.unl.edu. Acesso em maio de 2020.

NÓBREGA, R.S.; SANTIAGO, G.A.C.F. Tendência de temperatura na superfície domar nos oceanos atlântico e pacífico e variabilidade de precipitação em Pernambuco. Mercator, v. 13, n. 1, p. 107-118, 2014.

NÓBREGA, R. S.; SANTIAGO, G. A. C. F.; SOARES, D. B. Tendências do controle climático Oceânico sob a variabilidade temporal da precipitação no Nordeste do Brasil Revista Brasileira de Climatologia, v. 18, p.276-292 2016.

NORONHA, G. C.; HORA, M. A. G. M.; SILVA, L. P. Análise do Índice de Anomalia de Chuva para a Microbacia de Santa Maria/Cambiocó, RJ. Revista Brasileira de Meteorologia, v. 31, n. 1, p. 74-81, 2016.

PETRUngARO, A. C. N.; HORA. M. A. G. M. Avaliação das Secas Meteorológica e Hidrológica na Bacia Contribuinte à Barragem de Juturnaíba, Estado do Rio de Janeiro, Brasil. Anuário do Instituto de Geociências, v. 42 n. 4, p. 309-321, 2019.

SILVA, R.M.; SANTOS, C.A.G.; DOS SANTOS, J.Y.G. Evaluation and modeling of runoff and sediment yield for different land covers under simulated rain in a semiarid region of Brazil. Int. J. Sediment Res. v.33, n.2, p.117-125, 2018.

SILVA, F. F.; DOS SANTOS, F. A.; DOS SANTOS, J. M. Índice de Anomalia de Chuva (IAC) aplicado ao estudo das precipitações no município de Caridade, Ceará, Brasil. Revista Brasileira de Climatologia, v. 27, p. 426- 442, 2020. 
UNCCD. United Nations Convention to Combat Desertification. Desertification: the invisible frontline. 2. ed. França: UNCCD, 2014.

VERNER, D. Vozes do povo: impactos socioeconômicos da seca no Nordeste do Brasil. In: DE NYS, E.; ENGLE, N.L.; MAGALHÃES, A.R. (orgs.). Secas no Brasil: política e gestão proativas. Brasília, DF: Centro de Gestão e Estudos Estratégicos- CGEE; Banco Mundial, 2016. 292 p. 